\title{
Validación de los cuestionarios PAQ-C e IPAQ-A en niños/as en edad escolar
}

\author{
Validation of the PAQ-C and IPAQ-A questionnaires in school children \\ Carlos Martín-Belloㄹ, Germán Vicente-Rodríguez ${ }^{1,2,3,4}$, Jose A. Casajús ${ }^{2,3,4,5}$, Alejandro Gómez-Bruton ${ }^{1,2,3}$ \\ 1 Facultad de ciencias de la salud y del deporte. Universidad de Zaragoza. España. \\ 2 Grupo de investigación GENUD (Growth, Exercise, NUtrition and Development). Universidad de Zaragoza. España. \\ 3 Centro de Investigación Biomédica en Red de Fisiopatología de la Obesidad y Nutrición (CIBERObn). España. \\ 4 Instituto Agroalimentario de Aragón (IA2). España. \\ 5 Facultad de ciencias de la salud. Universidad de Zaragoza. España.
}

\author{
CORRESPONDENCIA: \\ Alejandro Gómez Bruton \\ bruton@unizar.es
}

Recepción: noviembre 2018• Aceptación: abril 2019
CÓMO CITAR EL ARTÍCULO:

Martín-Bello, C., Vicente-Rodríguez, G., Casajús, J. A., \& GómezBruton, A. (2020). Validación de los cuestionarios PAQ-C e IPAQ-A en niños/as en edad escolar. Cultura, Ciencia y Deporte, 15(44), 177-187.

\section{Resumen}

Los cuestionarios se han propuesto como una buena alternativa a los métodos objetivos (acelerometría o podometría), sin embargo, no parece existir un consenso sobre qué cuestionario es el mejor para cuantificar los niveles de actividad física en niños españoles. El objetivo del presente estudio fue el de validar los cuestionarios "Physical Activity Questionnaire for Children" (PAQ-C) y el "International Physical Activity Questionnaire for Adolescents" (IPAQ-A) completados por niños/as y sus padres. Para ello, 24 niños/as sanos $(9.1 \pm 0.6$ años) llevaron un acelerómetro siete días consecutivos, completando tanto ellos como sus padres el PAQ-C e IPAQ-A. La puntuación del PAQ-C se comparó con la actividad física (AF) moderada y vigorosa obtenida por el acelerómetro, mientras que en el IPAQ-A se compararon minutos por intensidades de AF con los del acelerómetro. Las correlaciones obtenidas entre el PAQ-C y acelerometría fueron positivas para niños/as ( $r h o=0.42 ; \mathrm{p}<.05$ ), aunque las tablas de contingencia mostraron diferencias entre métodos. Para el IPAQ-A las correlaciones con lo reportado por niños/as fueron bajas (Pearson(r) -0.36 a 0.31), mientras que las de los padres fueron ligeramente superiores. Se observaron diferencias significativas entre lo reportado en los cuestionarios frente a lo registrado por los acelerómetros. A partir del presente estudio se puede concluir que los cuestionarios PAQ-C e IPAQ-A tienen una validez cuestionable para cuantificar la $A F$ en niños/as españoles.

Palabras clave: Acelerometría, niños, validación, actividad física.

\section{Abstract}

Physical activity questionnaires have been proposed as useful alternatives of objective measures (accelerometry or podometry). Nonetheless, there is a lack of consensus regarding which questionnaire better quantifies physical activity levels in Spanish children. The main aim of the present study was to determine the validity of the "Physical Activity Questionnaire for Children" (PAQ-C) and the "International Physical Activity Questionnaire for Adolescents" (IPAQ-A) questionnaires completed both by children and their parents. Twenty-four healthy children $(9.1 \pm 0.6$ years) wore an accelerometer seven consecutive days. The children and their parents completed the PAQ-C and IPAQ-A questionnaires. The PAQ-C was compared to the moderate and vigorous physical activity obtained by the accelerometer, while the IPAQ-A was compared according to intensities of physical activity (PA). Correlations obtained between the PAQ-C and accelerometry were positive for children (rho=0.42;p<.05), although the contingency tables showed differences between methods. For the IPAQ-A the correlations between accelerometry and data reported by the children were low (Pearson(r) -0.36 to 0.31), while those reported by the parents were slightly higher. Significant differences were found between the reported data registered by the questionnaires and accelerometry data. To summarize, the PAQ-C and IPAQ-A questionnaires have a questionable validity when quantifying PA in Spanish children.

Key words: Accelerometry, children, validation, physical activity. 


\section{Introducción}

Niveles bajos de actividad física (AF) y sedentarismo se han visto asociados con la aparición de diferentes patologías y problemas de salud ( $\mathrm{Hu}, \mathrm{Li}$, Colditz, Willett, \& Manson, 2003; Warren et al., 2010). Se ha demostrado a través de numerosos estudios que la realización de AF de manera regular durante la infancia aporta beneficios sobre la composición corporal (Laguna, Ruiz, Lara, \& Aznar, 2013), salud ósea (GomezBruton et al., 2016; Lozano-Berges et al., 2018), salud psicológica (Stults-Kolehmainen \& Sinha, 2014) y capacidad cardiorrespiratoria (Loprinzi, Cardinal, Loprinzi, \& Lee, 2012; Mayorga-vega, Parra Saldías, \& Viciana, 2019) entre otras.

De este modo es necesario conocer con precisión los niveles de AF durante la infancia, además de validar y desarrollar instrumentos capaces de evaluar los niveles de AF en esta población escolar. Los cuestionarios autoadministrados proporcionan una forma conveniente de evaluar los patrones de actividad en grandes poblaciones (Hills, Mokhtar, \& Byrne, 2014), sin embargo, aunque existen multitud de cuestionarios para estimar los niveles de AF en niños (Martínez-Lemos, Ayán Pérez, Sánchez Lastra, Cancela Carral, \& Valcarce Sánchez, 2016), pocos estudios han evaluado la validez de dichos cuestionarios.

Respecto al uso de métodos objetivos para valorar los niveles de $\mathrm{AF}$, se ha observado que los acelerómetros cuantifican correctamente la AF realizada por niños en comparación con métodos de referencia como la calorimetría indirecta o el agua doblemente marcada (Ekelund et al., 2001). Por lo tanto, diversos estudios utilizan la acelerometría para evaluar la validez de los cuestionarios, comparando los minutos de $\mathrm{AF}$ reportada por el participante a través de los cuestionarios con los datos registrados por el acelerómetro (Lee, Macfarlane, Lam, \& Stewart, 2011).

Existen multitud de cuestionarios para estimar los niveles de $\mathrm{AF}$ en niños/as, entre los que destaca el Cuestionario de Actividad Física para Niños o Physical Activity Questionnaire for Children (PAQ-C) (Crocker, Bailey, Faulkner, Kowalski, \& McGrath, 1997), que ha demostrado tener una buena fiabilidad y validez en niños de 8 a 13 años (Janz, Lutuchy, Wenthe, \& Levy, 2008; Kowalski \& Crocker, 1997). Este mismo cuestionario ha sido evaluado recientemente en su versión española, obteniendo una alta fiabilidad pero una cuestionable validez para evaluar la AF total y la MVPA en niños y niñas españoles de $10.9 \pm 1.2$ años (BenítezPorres, López-Fernández, et al., 2016). En su estudio, Benítez-Porres et al. (2016) mencionan como posibles limitaciones la subjetividad inherente de reportar in- formación sobre un comportamiento o las dificultades de comprensión por parte de los niños.

Otro de los cuestionarios más empleados en poblaciones adolescentes es el International Physical Activity Questionnaire for Adolescents (IPAQ-A) creado por el estudio HELENA (Hagströmer et al., 2008). Este cuestionario es una versión adaptada del famoso cuestionario internacional IPAQ, el cual ha sido validado en numerosas ocasiones (Bortolozo, Santos, Pilatti, \& Canteri, 2017; Lee et al., 2011). Este cuestionario, reporta la $\mathrm{AF}$ en minutos por día en las diferentes intensidades, al igual que el acelerómetro. El IPAQ-A ha sido validado en población adolescente por el mismo estudio que lo creó, obteniendo una buena validez únicamente en participantes de 15-17 años, aunque en diferentes idiomas, entre ellos su versión en español. Los ítems de este último cuestionario, aunque está enfocado hacia adolescentes, preguntan sobre acciones de la vida cotidiana que también realizan los niños en edad escolar.

Por lo tanto, y tras evaluar la literatura existente, se presenta la necesidad de evaluar los resultados obtenidos por los cuestionarios PAQ-C e IPAQ-A completados por niños y padres, tratando de salvar la limitación de estudios previos de falta de comprensión por parte de los niños/as, con el objetivo de valorar la AF realizada en edad escolar. El PAQ-C fue seleccionado ya que está dirigido para una población muy concreta, niños de 8-12 años. Sin embargo, el PAQ-C tan solo cuantifica en una escala Likert de 1 a 5 , sin dividir en niveles de intensidad. Por lo tanto, también se utilizó IPAQ-A, ya que cuantifica la $\mathrm{AF}$ en minutos por intensidades de $\mathrm{AF}$, al igual que el acelerómetro por lo que podría ser un instrumento muy útil.

Consecuentemente, los objetivos del presente estudio son: 1) evaluar la validez de dos cuestionarios para la cuantificación de los niveles de actividad física en niños frente a acelerometría, y 2) comparar los resultados obtenidos en la comparación cuestionario vs. acelerómetro en función de la respuesta proporcionada por hijos o padres.

\section{Material y métodos}

\section{Comité ético}

Los niños y sus padres fueron convenientemente informados de las características del estudio, estos últimos firmaron un consentimiento informado para su participación. El estudio fue realizado de acuerdo con la Declaración de Helsinki, Finlandia en 1961 (revisada en Fortaleza, Brasil 2013). Además, fue aprobado 
por el Comité Ético de la Investigación de la Comunidad Autónoma de Aragón (CEICA, Acta No 09/2017) el 12 de mayo de 2017.

\section{Participantes}

En el presente estudio participaron 43 niños (17 chicos y 26 chicas) de entre 8 y 10 años, de un colegio de la ciudad de Zaragoza (Aragón, España). Se trata de una muestra de conveniencia, similar a estudios de validación de cuestionarios de actividad física (Kaleth, Ang, Chakr, \& Tong, 2010; Mäder, Martin, Schutz, \& Marti, 2006), buscando una muestra de 30 participantes.

Los criterios de inclusión para formar parte del estudio fueron: 1) cursando educación primaria, 2) edades comprendidas entre los 8 y los 12 años.

Se invitó a participar en la investigación a los cursos de $3^{\circ}$ y $4^{\circ}$ de Educación Primaria los cuales comprendían 3 clases por curso, aceptando participar 43 niños (17 chicos y 26 chicas) de un total de 146, con edades comprendidas entre los 8 y los 10 años.

Tras realizar el seguimiento por acelerometría y la recogida de cuestionarios se descartó a 10 de los participantes por no haber contestado a todos los cuestionarios correctamente y a 9 sujetos por no haber llevado durante los 7 días el acelerómetro. De este modo la muestra final fue de 24 participantes (10 chicos y 14 chicas), sobre la que se realizó el pertinente análisis de datos.

\section{Cuestionarios de actividad física utilizados}

El cuestionario PAQ-C (Kowalski \& Crocker, 1997) fue diseñado para estimar la actividad física en niños de 8 a 12 años en un estudio de la Universidad de Saskatchewan (Canadá), en el que se pregunta por su AF durante los distintos momentos del día, como clase de educación física, transporte y tiempo libre. Está formado por 10 preguntas que valoran distintos aspectos de los niveles de actividad física realizada por el niño mediante la escala Likert de 5 puntos, si bien solo se utilizan 9 preguntas para calcular la puntuación final. Se utilizó la versión en castellano utilizada por (Manchola, Bagur, \& Girabent, 2014).

El cuestionario IPAQ-A está formado por 11 preguntas divididas en cuatro bloques, actividad física en el colegio, tareas domésticas y del jardín, transporte y actividad física, y actividad física durante el tiempo de ocio. Estas 11 preguntas se diferencian sobre la intensidad de la actividad física que preguntan, una pregunta sobre sedentarismo, tres preguntas sobre $\mathrm{AF}$ ligera, cuatro preguntas sobre AF moderada y tres preguntas sobre AF vigorosa. Este cuestionario distingue
3 intensidades: ligera, moderada y vigorosa, similares a las intensidades proporcionadas por el acelerómetro. Se utilizó el cuestionario en español, que ya había sido traducido por el mismo estudio HELENA (Hagströmer et al., 2008) para su utilización.

\section{Acelerometría}

El acelerómetro utilizado en la investigación fue el GENEActiv Accelerometer (Activinsights Limited, Cambridge, UK), un pequeño acelerómetro triaxial de muñeca sumergible con una larga duración de batería. Por este motivo ha sido utilizado en numerosos estudios, siendo validado para población adulta (Esliger et al., 2011) e infantil (Phillips, Parfitt, \& Rowlands, 2013). El registro de datos se realiza en los 3 ejes, midiendo la intensidad de la actividad y los periodos de sueño y vigilia. Está equipado con un filtro que discrimina los movimientos humanos de las vibraciones. Los participantes llevaron el acelerómetro durante 7 días consecutivos en la muñeca izquierda, con una configuración previa de $30 \mathrm{~Hz}$.

La extracción de datos se realizó con el software informático de GENEActive. Para el análisis de estos datos extraídos se utilizó el programa informático $R$ Studio, con la configuración del paquete específico de acelerometría de (Marín Puyalto, Casajús Mallén, \& Ojeda Cabrera, 2017). En esta extracción de datos se agruparon los datos en epoch de $15 \mathrm{~s}$. Los puntos de corte empleados para la categorización de la AF fueron $85.5,141.3$ y 449.1gs para AF ligera, moderada y vigorosa respectivamente, los cuales fueron adaptados del estudio realizado por (Schaefer, Nigg, Hill, Lois, \& Browning, 2014) en población infantil.

\section{Protocolo de estudio}

En tandas de 15 niños/as, se entregó a cada participante un acelerómetro, dos cuestionarios para rellenar por los niños (PAQ-C y IPAQ-A) y estos dos mismos cuestionarios para completar por uno de los padres, junto con las instrucciones de utilización del acelerómetro y de cumplimentación de los cuestionarios. Siguiendo las instrucciones entregadas, al finalizar la semana debían cumplimentar los cuestionarios y devolverlos junto con los acelerómetros. La recogida de datos se llevó a cabo durante el mes de junio de 2017.

\section{Análisis estadístico}

Se realizó una recogida de datos de forma prospectiva. El análisis estadístico se llevó a cabo por medio del programa informático IBM SPSS Statistics 20. 
Tabla 1. Características de los participantes del estudio, y descriptivos de acelerometría y cuestionarios

\begin{tabular}{|c|c|c|c|}
\hline & Todos (N = 24) & Chicos $(n=10)$ & Chicas $(n=14)$ \\
\hline Edad (años) & $9.08 \pm 0.58$ & $8.90 \pm 0.57$ & $9.21 \pm 0.58$ \\
\hline \multicolumn{4}{|l|}{ ACELEROMETRÍA } \\
\hline Sedentarismo (min/día) & $1280.27 \pm 40.94$ & $1269.73 \pm 37.39$ & $1287.80 \pm 43.02$ \\
\hline AF ligera (min/día) & $90.29 \pm 17.76$ & $88.01 \pm 14.39$ & $91.92 \pm 20.19$ \\
\hline AF moderada (min/día) & $62.23 \pm 21.87$ & $73.97 \pm 23.51$ * & $53.84 \pm 16.78^{*}$ \\
\hline AF vigorosa (min/día) & $5.34 \pm 4.16$ & $8.19 \pm 4.71 *$ & $3.30 \pm 2.14^{*}$ \\
\hline MVPA (min/día) & $67.56 \pm 25.38$ & $82.16 \pm 27.40 *$ & $57.14 \pm 18.40$ * \\
\hline AF Total (min/día) & $157.86 \pm 36.55$ & $170.17 \pm 37.36$ & $149.06 \pm 34.60$ \\
\hline \multicolumn{4}{|l|}{ IPAQ-A NIÑOS/AS } \\
\hline Sedentarismo IPAQ-A (min/día) & $20.11 \pm 28.10$ & $14.14 \pm 18.18$ & $24.37 \pm 33.49$ \\
\hline AF ligera IPAQ-A (min/día) & $78.42 \pm 79.17$ & $80.29 \pm 47.56$ & $77.92 \pm 97.57$ \\
\hline AF moderada IPAQ-A (min/día) & $50.59 \pm 44.28$ & $52.61 \pm 44.91$ & $49.14 \pm 45.47$ \\
\hline AF vigorosa IPAQ-A (min/día) & $55.90 \pm 50.95$ & $67.83 \pm 42.27$ & $47.39 \pm 56.24$ \\
\hline MVPA IPAQ-A (min/día) & $106.49 \pm 88.25$ & $120.44 \pm 78.07$ & $96.53 \pm 96.45$ \\
\hline AF Total IPAQ-A (min/día) & $184.92 \pm 148.44$ & $200.73 \pm 108.77$ & $173.62 \pm 174.54$ \\
\hline \multicolumn{4}{|l|}{ IPAQ-A PADRES } \\
\hline Sedentarismo IPAQ-A (min/día) & $19.15 \pm 21.12$ & $16.43 \pm 18.56$ & $21.10 \pm 23.25$ \\
\hline AF ligera IPAQ-A (min/día) & $65.21 \pm 58.85$ & $65.93 \pm 43.30$ & $64.69 \pm 69.49$ \\
\hline AF moderada IPAQ-A (min/día) & $42.08 \pm 34.07$ & $48.71 \pm 30.31$ & $37.34 \pm 36.88$ \\
\hline AF vigorosa IPAQ-A (min/día) & $47.85 \pm 39.20$ & $57.79 \pm 43.71$ & $40.75 \pm 35.57$ \\
\hline MVPA IPAQ-A (min/día) & $89.93 \pm 58.66$ & $106.50 \pm 56.14$ & $78.09 \pm 59.53$ \\
\hline AF Total IPAQ-A Padres (min/día) & $155.14 \pm 93.62$ & $172.43 \pm 81.25$ & $142.79 \pm 102.68$ \\
\hline \multicolumn{4}{|l|}{ PAQ-C NIÑOS/AS } \\
\hline AF puntuación total (media) & $2.72 \pm 0.60$ & $2.72 \pm 0.66$ & $2.71 \pm 0.58$ \\
\hline \multicolumn{4}{|l|}{ PAQ-C PADRES } \\
\hline AF puntuación total (media) & $2.60 \pm 0.63$ & $2.66 \pm 0.61$ & $2.56 \pm 0.67$ \\
\hline
\end{tabular}

Escala PAQ-C (1-5) AF = Actividad Física, MVPA = Actividad Física de Moderada a Vigorosa, ${ }^{*}$ diferencias significativas entre chicos y chicas ( $\mathrm{p}<0.05$ ).

Para comparar la actividad física realizada por niños y niñas (tanto la subjetiva (cuestionarios) como la objetiva (acelerometría)) se utilizaron pruebas $\mathrm{T}$ de student para muestras independientes. Para comparar lo reportado por niño/as o por padres con la $\mathrm{AF}$ registrada a través de los acelerómetros se utilizaron correlaciones de Pearson (IPAQ-A vs. Acelerometria) y Spearman (PAQ-C vs. acelerometría), pruebas $\mathrm{T}$ de Student para muestras dependientes (IPAQ-A vs. Acelerometría).

Además, se evaluó la concordancia entre el PAQ-C y la acelerometría mediante tablas de contingencia con el índice Kappa. Para ello, se utilizaron los valores reportados por (Benítez-Porres, Alvero-Cruz, Sardinha, López-Fernández, \& Elvis A, 2016), donde se determina que un resultado en el PAQ-C superior a 2.73 equivale a más de 60 minutos al día de MVPA. Además, se utilizaron correlaciones intraclase según los puntos de corte de (Atkinson \& Nevill, 1998), que establece los valores de 0.7 a 0.8 en cuestionable, $\mathrm{y}>0.9$ como una correlación alta. Por último, se efectuaron gráficos de Bland-Altman con el objetivo de visualizar la homogeneidad y heterocedasticidad de los cuestionarios por medio del programa informático SigmaPlot 10.0.

\section{Resultados}

\section{Descriptivos}

Para los datos de actividad física registrada mediante acelerometría, los chicos mostraron mayor tiempo en $\mathrm{AF}$ moderada $(73.97 \pm 23.51$ minutos chicos vs. $53.84 \pm 16.78$ minutos chicas), AF vigorosa (8.19 \pm 4.71 minutos chicos vs. $3.30 \pm 2.14$ minutos chicas) y MVPA (82.16 \pm 27.40 minutos chicos vs. $57.14 \pm$ 18.40 minutos chicas) que las chicas. Mientras que los cuestionarios IPAQ-A y PAQ-C no detectaron diferencias significativas entre sexos (Tabla 1 ).

\section{Acelerometría vs. IPAQ-A}

Como se muestra en la Tabla 2, no se observó ninguna correlación significativa entre la AF registrada por el acelerómetro y la reportada por los niños/as. Sin embargo, cuando el cuestionario fue completado por los padres sí que se observaron correlaciones significativas en el caso de la AF moderada y la MVPA ( $r=0.54$ y 0.56 , ambas $\mathrm{p}<0.01$ ).

Se observó que el cuestionario infraestima el tiempo de AF moderada cuando lo completan los padres, y so- 
Tabla 2. Correlaciones entre acelerometría y el cuestionario IPAQ-A.

\begin{tabular}{|c|c|c|c|c|c|}
\hline & $\begin{array}{l}\text { AF ligera } \\
\text { (min/día) }\end{array}$ & $\begin{array}{l}\text { AF moderada } \\
\text { ( } \mathrm{min} / \mathrm{día})\end{array}$ & $\begin{array}{l}\text { AF vigorosa } \\
\text { (min/día) }\end{array}$ & $\begin{array}{c}\text { MVPA } \\
\text { (min/día) }\end{array}$ & $\begin{array}{l}\text { AF total } \\
\text { (min/día) }\end{array}$ \\
\hline NIÑOS/AS IPAQ-A & & Datos acelero & & & \\
\hline AF ligera & -0.36 & & & & \\
\hline AF moderada & & 0.21 & & & \\
\hline AF vigorosa & & & 0.26 & & \\
\hline MVPA & & & & 0.31 & \\
\hline AF total & & & & & 0.02 \\
\hline \multicolumn{6}{|l|}{ PADRES IPAQ-A } \\
\hline AF ligera & -0.27 & & & & \\
\hline AF moderada & & $0.54 * *$ & & & \\
\hline AF vigorosa & & & 0.27 & & \\
\hline MVPA & & & & $0.56 * *$ & \\
\hline AF total IPAQ-A & & & & & 0.28 \\
\hline
\end{tabular}

$\mathrm{AF}=$ Actividad Física, MVPA = Actividad Física de Moderada a Vigorosa, ${ }^{*} p<0.05{ }^{* *} p<0.01$.

Tabla 3. Diferencias entre acelerometría e IPAQ-A.

\begin{tabular}{|c|c|c|c|}
\hline & \multirow{2}{*}{$\begin{array}{c}\text { Diferencia media } \\
\text { (Acelerometría - Cuestionario) }\end{array}$} & \multicolumn{2}{|c|}{ IC 95\% } \\
\hline & & Inferior & Superior \\
\hline AF ligera (min/día) & $11.87 \pm 87.22$ & -24.96 & 48.70 \\
\hline AF moderada (min/día) & $11.64 \pm 44.99$ & -7.36 & 30.64 \\
\hline AF vigorosa (min/día) & $-50.56 \pm 50.01 *$ & -71.68 & -29.45 \\
\hline MVPA (min/día) & $-38.93 \pm 83.86 *$ & -74.34 & -3.52 \\
\hline AF total (min/día) & $-27.06 \pm 152.19$ & -91.32 & 37.20 \\
\hline \multicolumn{4}{|l|}{ PADRES } \\
\hline AF ligera (min/día) & $25.08 \pm 65.86$ & -2.73 & 52.89 \\
\hline AF moderada (min/día) & $20.15 \pm 28.98^{*}$ & 7.91 & 32.39 \\
\hline AF vigorosa (min/día) & $-42.51 \pm 38.29 *$ & -58.68 & -26.34 \\
\hline MVPA (min/día) & $-22.36 \pm 49.22 *$ & -43.15 & -1.58 \\
\hline AF total (min/día) & $2.72 \pm 90.51$ & -35.50 & 40.94 \\
\hline
\end{tabular}

$\mathrm{AF}=$ Actividad Física, MVPA = Actividad Física de Moderada a Vigorosa, ${ }^{*} \mathrm{p}<0.05$.

breestima la AF vigorosa y MVPA independientemente de si lo completan los niños o los padres (Tabla 3). Sin embargo, no se observaron diferencias significativas ( $p>0.05$ ) entre el tiempo medido con el acelerómetro y el estimado por el cuestionario para el resto de intensidades (Tabla 3). Se pude observar en la Tabla 4 que todas las correlaciones intraclase entre lo registrado por el acelerómetro y lo reportado mediante el cuestionario IPAQ-A fueron bajas.

En la Figura 1 se presentan los gráficos Bland-Altman para la comparativa entre lo reportado por los niños y lo registrado por el acelerómetro, mientras que en la Figura 2 se presentan los datos de la comparativa entre lo reportado por los padres y lo registrado por el acelerómetro. Se puede observar que en todos los gráficos los datos presentan heterocedasticidad, ya que cuando la $\mathrm{AF}$ realizada es más baja el cuestionario la infraestimaba, mientras que cuando era mayor, se sobreestimaba su realización a través del cuestionario IPAQ-A para todas las intensidades a excepción de la $\mathrm{AF}$ vigorosa, para la que siempre se sobreestima la $\mathrm{AF}$ reportada mediante cuestionario.

\section{Acelerometría vs. PAQ-C}

Se observaron correlaciones significativas $(\mathrm{p}<0.05)$ entre la acelerometría y lo reportado por niños/as en el caso de la AF moderada y la MVPA (Tabla 5). También se encontraron correlaciones significativas entre el cuestionario PAQ-C contestado por los padres y la AF moderada medida por acelerometría.

En las Tablas 6 y 7 se presentan las tablas de contingencia comparando la clasificación que proporciona el acelerómetro (punto de corte 60 minutos al día de MVPA), frente a datos proporcionados por el cuestionario (punto de corte 2,73 propuesto por 
Benítez-Porres et al que corresponde a 60 minutos al día de MVPA). Se obtuvieron valores de Kappa de 0,33 $(\mathrm{p}=0,11)$ y $0,26(\mathrm{p}=0,18)$ para hijos y padres respectivamente. Estos valores indican una asociación débil entre métodos (Landis \& Koch, 1977). Por lo que se puede afirmar que la acelerometría y el cuestionario PAQ-C no cuantificaron y clasificaron la actividad física de la misma manera.

Niños/as VS. Padres

Se encontraron diferencias significativas entre lo reportado por padres e hijos tanto para el PAQ-C (Tabla 8; $\mathrm{p}<0,05)$, como para todas las intensidades del IPAQ-A (Tabla 9; todas $\mathrm{p}<0,05)$. En el caso del PAQ-C, comparando por medio de Bland-Altman, en las respuestas de niños/as y padres (Figura 3) se observa que los niveles de actividad no afectaban a la diferencia de respuesta entre niños y padres.

\section{Discusión}

El principal hallazgo del presente estudio es que ninguno de los dos cuestionarios evaluados estimó correctamente los niveles de actividad física registrados mediante acelerometría, aunque el PAQ-C completado por los niños/as mostró correlaciones significativas con la MVPA reportada por el acelerómetro.

La falta de consenso entre métodos podría deberse a la propia naturaleza de los mismos, ya que el acelerómetro (GENEActive), considerado como un método válido para realizar la validación (Ekelund et al., 2001), proporciona información sobre el movimiento corporal, mientras que los cuestionarios a menudo piden a los encuestados que cuantifiquen el esfuerzo o la frecuencia de las actividades realizadas. Además, los acelerómetros solo pueden proporcionar información sobre la aceleración, no proporcionando información correcta sobre la intensidad de las actividades (Hendelman, Miller, Baggett, Debold, \& Freedson, 2000), como llevar cargas pesadas, subir las escaleras, o el registro de otras actividades como skateboarding, ciclismo o natación sobre las que se preguntan en el primer ítem del PAQ-C.

Para la extracción de datos se utilizaron puntos de corte para la AF ligera, moderada y vigorosa en niños y adolescentes del estudio de Schaefer et al., (2014). Es posible que otros puntos de corte hubieran arrojado resultados diferentes, cuantificando las intensidades de $\mathrm{AF}$ de diferente modo. No obstante, las asociaciones y diferencias con AF total continuarían siendo las mismas porque esta variable no depende en gran medida de los valores de corte seleccionados.
Tabla 4. Correlación Intraclase (ICC) entre acelerómetro e IPAQ-A.

\begin{tabular}{ll}
\hline NIÑOS/AS & ICC \\
\hline AF ligera (min/día) & -0.38 \\
AF moderada (min/día) & 0.28 \\
AF vigorosa (min/día) & 0.42 \\
MVPA (min/día) & 0.25 \\
AF total (min/día) & 0.02 \\
\hline PADRES & \\
\hline AF ligera (min/día) & -0.30 \\
AF moderada (min/día) & 0.57 \\
AF vigorosa (min/día) & 0.05 \\
MVPA (min/día) & 0.54 \\
AF total (min/día) & 0.33 \\
\hline
\end{tabular}

AF = Actividad Física, MVPA = Actividad Física de Moderada a Vigorosa valores de 0.7 a 0.8 cuestionable, y $>0.9$ correlación alta.

Tabla 5. Correlaciones entre acelerometría y el cuestionario PAQ-C.

\begin{tabular}{lcc}
\hline & $\begin{array}{c}\text { PAQ-C niños/as } \\
\text { (media) }\end{array}$ & $\begin{array}{c}\text { PAQ-C padres } \\
\text { (media) }\end{array}$ \\
\hline AF ligera (min/día) & 0.18 & 0.22 \\
AF moderada (min/día) & $0.44^{*}$ & $0.41^{*}$ \\
AF vigorosa (min/día) & 0.24 & 0.22 \\
MVPA (min/día) & $0.42^{*}$ & 0.38 \\
AF total (min/día) & 0.30 & 0.30 \\
\hline
\end{tabular}

$\mathrm{AF}=$ Actividad Física, MVPA = Actividad Física de Moderada a Vigorosa ${ }^{*} p<0.05 * * p<0.01$.

Tabla 6. Tablas de contingencia entre acelerometría y PAQ-C niños/as.

\begin{tabular}{lcc}
\hline & \multicolumn{2}{c}{ Datos cuestionario } \\
\cline { 2 - 3 } Datos acelerometría & $\begin{array}{c}\text { PAQ-C } \\
\text { (media) }<2.73\end{array}$ & $\begin{array}{c}\text { PAQ-C } \\
\text { (media) }>2.73\end{array}$ \\
\hline MVPA (min/día) $<60 \min$ & $7(29.2 \%)$ & $4(16.7 \%)$ \\
MVPA (min/día) $>60 \min$ & $4(16.7 \%)$ & $9(37.5 \%)$ \\
\hline
\end{tabular}

MVPA = Actividad Física de Moderada a Vigorosa

Tabla 7. Tablas de contingencia entre acelerometría y PAQ-C padres.

\begin{tabular}{lcc}
\hline & \multicolumn{2}{c}{ Datos cuestionario } \\
\cline { 2 - 3 } Datos acelerometria & PAQ-C (media) & PAQ-C (media) \\
& $<2.73$ & $>2.73$ \\
\hline MVPA (min/día) $<60 \min$ & $8(33.3 \%)$ & $3(12.5 \%)$ \\
MVPA (min/día) $>60 \min$ & $6(25.0 \%)$ & $7(29.2 \%)$ \\
\hline
\end{tabular}

MVPA = Actividad Física de Moderada a Vigorosa.

Tabla 8. Prueba T-student entre PAQ-C niños/as vs. PAQ-C padres.

\begin{tabular}{lccc}
\hline & Diferencia media & \multicolumn{2}{c}{ IC 95\% } \\
& (niños - padres) & Inferior & Superior \\
\hline AF ligera (min/día) & $0.12 \pm 0.30 *$ & -0.01 & 0.24 \\
\hline
\end{tabular}

${ }^{*} p<0.05$ 
Tabla 9. Prueba T-student entre IPAQ-A niños vs. IPAQ-A padres.

\begin{tabular}{|c|c|c|c|}
\hline & \multirow{2}{*}{$\begin{array}{l}\text { Diferencia media } \\
\text { (niños - padres) }\end{array}$} & \multicolumn{2}{|c|}{ IC $95 \%$} \\
\hline & & Inferior & Superior \\
\hline AF ligera IPAQ-A & $13.21 \pm 37.76^{*}$ & -2.73 & -2.73 \\
\hline AF moderada IPAQ-A & $8.51 \pm 43.03^{*}$ & -9.66 & -9.66 \\
\hline AF vigorosa IPAQ-A & $8.05 \pm 33.04 *$ & -5.90 & -5.90 \\
\hline MVPA IPAQ-A & $16.56 \pm 60.08^{*}$ & -8.80 & -8.80 \\
\hline AF total IPAQ-A & $29.78 \pm 88.54^{*}$ & -7.61 & -7.61 \\
\hline
\end{tabular}

$\mathrm{AF}=$ Actividad Física, MVPA = Actividad Física de Moderada a Vigorosa, ${ }^{*} \mathrm{p}<0.05$.

Las mediciones del acelerómetro se realizaron durante el mismo período de tiempo al que se refiere el cuestionario. Por lo tanto, no hay ninguna razón para creer que los encuestados no se refirieron a la misma semana que fue medida por el acelerómetro.

En cuanto al cuestionario IPAQ-A, los resultados obtenidos son llamativos, ya que este cuestionario ha sido validado en adolescentes en el estudio HELENA (Hagströmer et al., 2008), por el cual fue creado. Esta validación solamente encontró correlaciones significativas entre acelerometría y cuestionario en adolescentes mayores (15-17 años), en edades de los 12 a los 15 años las correlaciones encontradas fueron bajas, con diferencias significativas entre ambos métodos. Estas bajas correlaciones cuando la AF era reportada por los niños podrían deberse a que los conceptos utilizados quizá no serían tan fácilmente comprendidos e interpretados por los niños, los cuales podrían tener dificultades para informar sobre actividades físicas, como ya se ha comprobado anteriormente (Caspersen, Nixon, \& DuRant, 1998). Como los adolescentes mayores (15-17 años) suelen realizar más actividades estructuradas, como deportes serían más fáciles de cuantificar en el cuestionario.

Para el PAQ-C se encontraron resultados similares a los encontrados para el IPAQ-A y similares también a los de estudios previos. El cuestionario PAQ-C ya fue validado desde su creación por (Kowalski \& Crocker, 1997), el cual obtuvo unas buenas correlaciones comparándolo con acelerometría, aunque esas correlaciones no fueron superiores a $r=0.49$. En otro estudio en el que se comparó con acelerometría uniaxial no se observaron correlaciones significativas, aunque sí lo hizo la versión similar para adolescentes PAQ-A, por lo que nuevamente podría tener su razonamiento en que los adolescentes tienen una mejor comprensión del cuestionario que los niños (Janz et al., 2008). En el caso del estudio de validación de la versión en español del PAQ-C de Benítez-Porres, López-Fernández, et al., (2016) tampoco se obtuvieron correlaciones significativas.

En el presente estudio se obtuvieron correlaciones significativas en el caso de la AF moderada y la MVPA cuando el cuestionario era contestado por los niños/ as. En todos los estudios de validación del PAQ-C la correlación que indicaba si el cuestionario tenía validez o no era la MVPA (Benítez-Porres, López-Fernández, et al., 2016; Janz et al., 2008; Kowalski \& Crocker, 1997), de este modo podríamos decir que los resultados de nuestro estudio muestran que el PAQ-C en su versión en español estima correctamente la MVPA si es completado por los niños. Sin embargo, cuando se compararon ambos métodos a través del índice de Kappa, se observó que el cuestionario no clasificaba bien en función del nivel de actividad. Estos resultados se podrían explicar por las dificultades de comprensión por parte de los niños para entender todos los ítems o ser conscientes de la AF que realizan. Además, se trata de un cuestionario que utiliza una escala Likert de 1 a 5 para cuantificar la MVPA realizada, pero no contempla la cuantificación del resto de intensidades de AF, por lo que su utilidad en el campo de la investigación es limitada.

Una novedad de este estudio es que en previsión de que los niños tuvieran problemas de interpretación, como se ha reportado previamente (Martínez-Lemos et al., 2016), y por tanto no reportasen adecuadamente la $\mathrm{AF}$, se registró y comparó la AF reportada por hijos/as y padres en cada cuestionario.

Cuando se comparó lo reportado por padres e hijos con acelerometría en el cuestionario IPAQ-A se obtuvieron valores similares, aunque existiendo correlaciones significativas entre AF moderada y MVPA en el caso de los padres. La explicación podría radicar en que los padres comprendieron mejor sobre lo que se preguntaba en cada uno de los ítems. Por tanto, la respuesta de los padres en el IPAQ-A cuantificaría mejor la AF que la reportada por los hijos. No obstante, se observaron diferencias significativas entre ambos métodos, ya que el cuestionario sobreestimaba la AF moderada e infraestimaba la MVPA respecto al acelerómetro. Sin embargo, en la comparación entre padres e hijos en el PAQ-C, los niños/as cuantificaron mejor la MVPA. Esto podría explicarse porque podrían ser más conscientes de la AF que realizan. Futuros estu- 


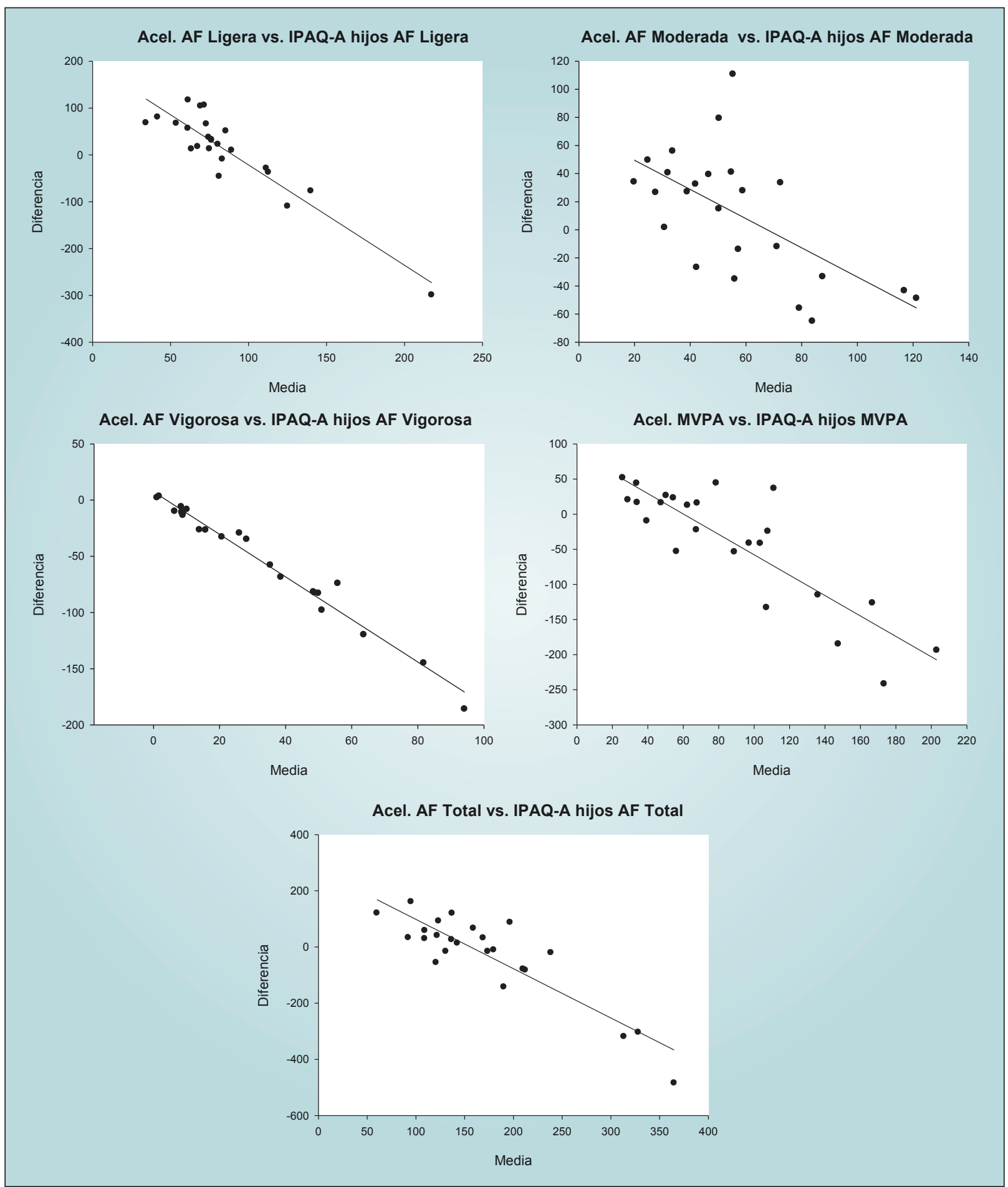

Figura 1. Bland-Altman acelerometría vs IPAQ-A niños.

dios deberían evaluar las diferencias encontradas entre padres e hijos a la hora de reportar la actividad física de los segundos, tratando de determinar qué grupo reporta mejor la actividad física realizada.

Aunque este estudio presenta diversas fortalezas, como son la utilización de acelerómetros para la validación de cuestionarios, la evaluación de la validez de dos cuestionarios de manera paralela completados por los mismos participantes, o también la cumplimentación de estos cuestionarios por parte de padres e hijos para comparar las diferencias, no está exento de limitaciones.

Una de las principales limitaciones de este estudio radica en el tamaño de la muestra, que ha sido algo inferior al de otros estudios de validación originales 


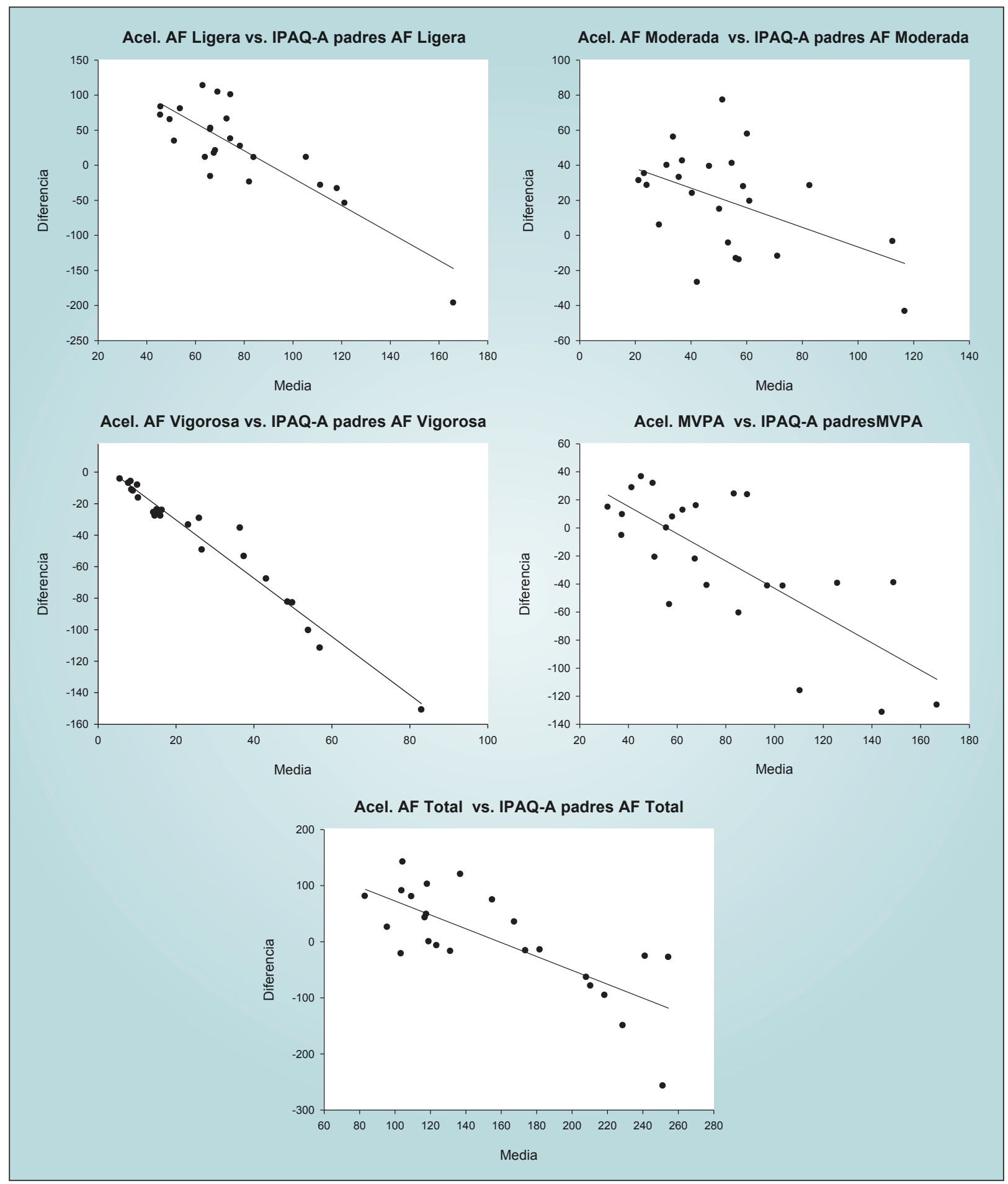

Figura 2. Bland-Altman acelerometría vs IPAQ-A padres.

(Hagströmer et al., 2008; Kowalski \& Crocker, 1997). Aunque en un primer momento la muestra a la que se accedió era mayor, la muestra final sobre la que se realizó el análisis estadístico fue inferior a lo que se habría deseado. Se señalaron algunos problemas como los errores en el recuerdo o tergiversaciones en el recuerdo que serían importantes cuando se trata de ni- ños (Sirard \& Pate, 2001). Estos sesgos se trataron de subsanar obteniendo también los cuestionarios completados por los padres, aunque no se controló si era el padre o la madre el que respondía a los mismos.

Futuras líneas de investigación podrían evaluar si existen diferencias entre lo reportado por padre, madre, niño y acelerómetro. 


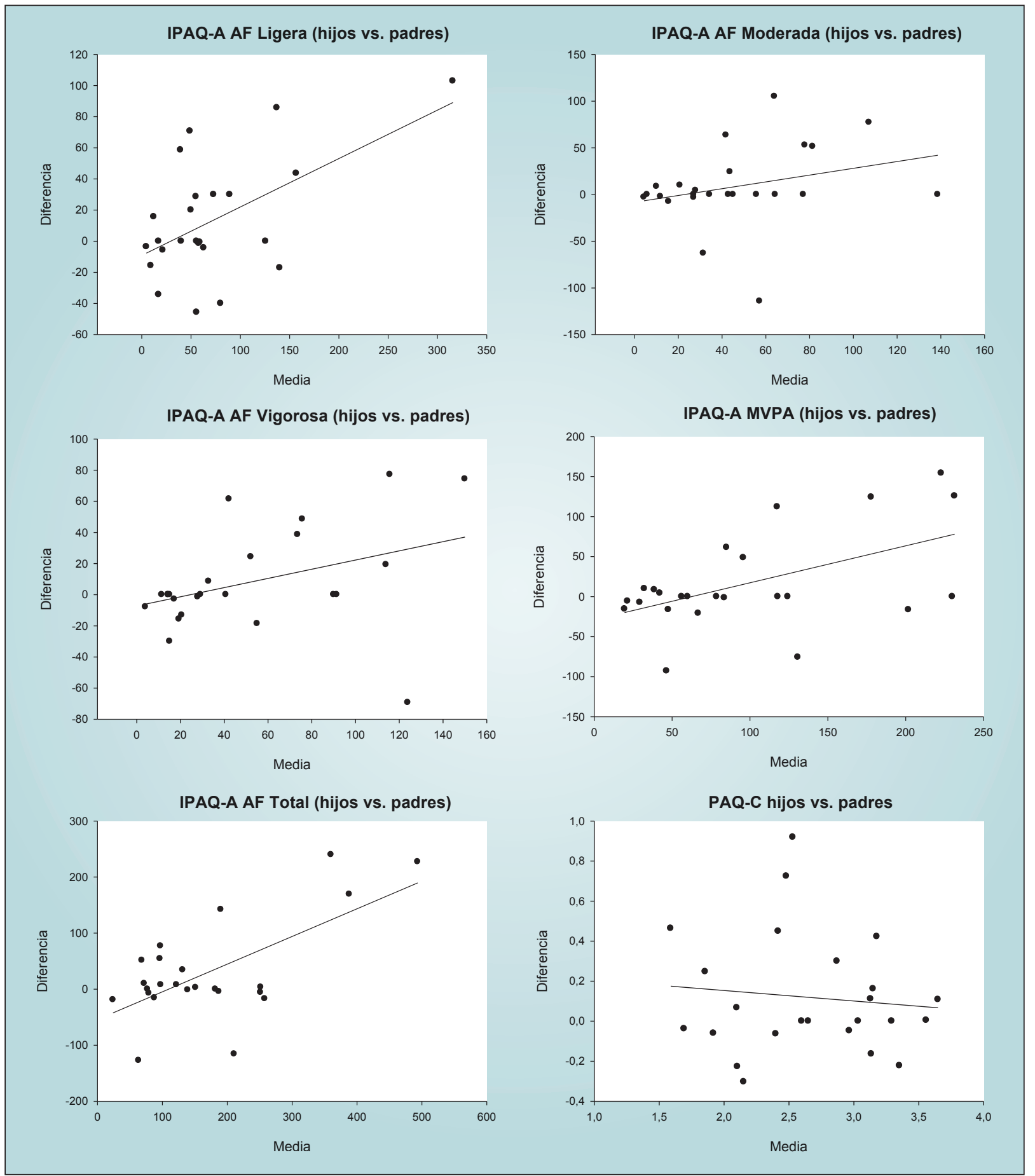

Figura 3. Bland-Altman IPAQ-A y PAQ-C, niños/as vs. padres.

\section{Conclusiones}

Los cuestionarios PAQ-C e IPAQ-A proporcionan información que no se correlaciona con la obtenida por acelerometría, por lo que parecen no ser válidos para cuantificar los niveles de actividad física en niños de 8 a 10 años independientemente de quién los complete (el niño/a o el padre/madre).
Lo reportado por los niños/as es diferente a lo que reportan los padres. Por lo tanto, estos cuestionarios no son apropiados para estimar los niveles de $\mathrm{AF}$ en población infantil de 8 a 10 años.

Futuros estudios deberían evaluar la validez de otros cuestionarios con el objetivo de poder cuantificar los niveles de AF en esta población sin necesidad de utilizar otros instrumentos más costosos. 


\section{B I B LIO G RAFÍA}

Atkinson, G., \& Nevill, A. (1998). Statistical Methods for Assssing Measurement Error (Reliability) in Variables Relevant to Sports Medicine. Sports Medicine, 26(4), 217-238. https://doi.org/10.2165/00007256199826040-00002

Benítez-Porres, J., Alvero-Cruz, J. R., Sardinha, L. B., López-Fernández, I., \& Elvis A, C. (2016). Cut-off values for classifying active children and adolescents using the Physical Activity Questionnaire: PAQ-C and PAQ-A. Nutr. Hosp., 33(5), 1108-1115. https://doi.org/10.20960/ nh. 574

Benítez-Porres, J., López-Fernández, I., Raya, J. F., Álvarez Carnero, S., Alvero-Cruz, J. R., \& Álvarez Carnero, E. (2016). Reliability and Validity of the PAQ-C Questionnaire to Assess Physical Activity in Children. Journal of School Health, 86(9), 677-685. https://doi. org/10.1111/josh.12418

Bortolozo, E. A. F. Q., Santos, C. B., Pilatti, L. A., \& Canteri, Y. G. (2017). Validez del cuestionario internacional de actividad física por correlación con podómetro. Rev.Int.Med.Cienc.Act.Fís.Deporte, 17(66), 397414. https://doi.org/10.15366/rimcafd2017.66.012

Caspersen, C. J., Nixon, P. A., \& DuRant, R. H. (1998). Physical activity epidemiology applied to children and adolescents. Exercise and Sport Sciences Reviews, 26, 341-403. Retrieved from http://www.ncbi.nlm. nih.gov/pubmed/9696995

Crocker, P. R., Bailey, D. A., Faulkner, R. A., Kowalski, K. C., \& McGrath, R. (1997). Measuring general levels of physical activity: preliminary evidence for the Physical Activity Questionnaire for Older Children. Med Sci Sports Exerc, 29(10), 1344-1349. https://doi. org/10.1097/00005768-199710000-00011

Ekelund, U., Sjöström, M., Yngve, A., Poortvliet, E., Nilsson, A., Froberg, K., ... Westerterp, K. (2001). Physical activity assessed by activity monitor and doubly labeled water in children. Medicine and Science in Sports and Exercise, 33(2), 275-81. https://doi. org/10.1097/00005768-200102000-00017

Esliger, D. W., Rowlands, A. V., Hurst, T. L., Catt, M., Murray, P., \& Eston, R. G. (2011). Validation of the GENEA accelerometer. Medicine and Science in Sports and Exercise, 43(6), 1085-1093. https://doi. org/10.1249/MSS.0b013e31820513be

Gomez-Bruton, A., Montero-Marín, J., González-Agüero, A., GarcíaCampayo, J., Moreno, L. A., Casajús, J. A., \& Vicente-Rodríguez, G. (2016). The Effect of Swimming During Childhood and Adolescence on Bone Mineral Density: A Systematic Review and Meta-Analysis. Sports Medicine, 46(3), 365-379. https://doi.org/10.1007/s40279015-0427-3

Hagströmer, M., Bergman, P., De Bourdeaudhuij, I., Ortega, F., Ruiz, J., Manios, Y., ... Sjöström, M. (2008). Concurrent validity of a modified version of the International Physical Activity Questionnaire (IPAQ-A) in European adolescents: The HELENA Study. International Journal of Obesity, 32, 42-48. https://doi.org/10.1038/ijo.2008.182

Hendelman, D., Miller, K., Baggett, C., Debold, E., \& Freedson, P. (2000). Validity of accelerometry for the assessment of moderate intensity physical activity in the field. Medicine and Science in Sports and Exercise, 32(9 Suppl), S442-9. https://doi.org/10.1097/00005768200009001-00002

Hills, A. P., Mokhtar, N., \& Byrne, N. M. (2014). Assessment of physical activity and energy expenditure: an overview of objective measures. Frontiers in Nutrition, 1, 5. https://doi.org/10.3389/fnut.2014.00005

Hu, F. B., Li, T. Y., Colditz, G. A., Willett, W. C., \& Manson, J. E. (2003). Television watching and other sedentary behaviors in relation to risk of obesity and type 2 diabetes mellitus in women. JAMA, 289(14), 1785-91. https://doi.org/10.1001/jama.289.14.1785

Janz, K. F., Lutuchy, E. M., Wenthe, P., \& Levy, S. M. (2008). Measuring activity in children and adolescents using self-report: PAQ- $C$ and PAQ-A. Medicine and Science in Sports and Exercise, 40(4), 767-72. https://doi.org/10.1249/MSS.0b013e3181620ed1

Kaleth, A. S., Ang, D. C., Chakr, R., \& Tong, Y. (2010). Validity and reliability of community health activities model program for seniors and short-form international physical activity questionnaire as physical activity assessment tools in patients with fibromyalgia. Disability and Rehabilitation, 32(5), 353-359. https://doi.org/10.3109/ 09638280903166352

Kowalski, K., \& Crocker, P. (1997). Validation of the physical activity questionnaire for older children. Pediatric Exercise. https://doi.org/ https://doi.org/10.1123/pes.9.2.174

Laguna, M., Ruiz, J. R., Lara, M. T., \& Aznar, S. (2013). Recommended levels of physical activity to avoid adiposity in Spanish children. Pediatric Obesity, 8(1), 62-69. https://doi.org/10.1111/j.20476310.2012.00086.x

Landis, J. R., \& Koch, G. G. (1977). The Measurement of Observer Agreement for Categorical Data. Biometrics, 33(1), 159. https://doi. org $/ 10.2307 / 2529310$

Lee, P. H., Macfarlane, D. J., Lam, T. H., \& Stewart, S. M. (2011). Validity of the international physical activity questionnaire short form ( IPAQ-SF ): A systematic review. International Journal of Behavioral Nutrition and Physical Activity, 8(1), 115. https://doi.org/10.1186/14795868-8-115

Loprinzi, P. D., Cardinal, B. J., Loprinzi, K. L., \& Lee, H. (2012). Benefits and Environmental Determinants of Physical Activity in Children and Adolescents. Obesity Facts, 5(4), 597-610. https://doi. org/10.1159/000342684

Lozano-Berges, G., Matute-Llorente, Á., González-Agüero, A., GómezBruton, A., Gómez-Cabello, A., Vicente-Rodríguez, G., \& Casajús, J. A. (2018). Soccer helps build strong bones during growth: a systematic review and meta-analysis. European Journal of Pediatrics, 177(3), 295-310. https://doi.org/10.1007/s00431-017-3060-3

Mäder, U., Martin, B. W., Schutz, Y., \& Marti, B. (2006). Validity of Four Short Physical Activity Questionnaires in Middle-Aged Persons. Medicine \& Science in Sports \& Exercise, 38(7), 1255-1266. https://doi. org/10.1249/01.mss.0000227310.18902.28

Manchola, J., Bagur, C., \& Girabent, M. (2014). Fiabilidad De La Versión Española Del Cuestionario De Actividad Física Paq-C. Revista Internacional de Medicina y Ciencias de La Actividad Física y El Deporte, $X(\mathrm{x})$, 1-13. https://doi.org/http://doi.org/10.15366/rimcafd2017.65.010

Marín Puyalto, J., Casajús Mallén, J. A., \& Ojeda Cabrera, J. L. (2017). Desarrollo de un software libre original para el análisis de datos de acelerometría. Universidad de Zaragoza. Retrieved from https://deposita.unizar.es/record/34220?ln=es\#

Martínez-Lemos, R., Ayán Pérez, C., Sánchez Lastra, A., Cancela Carral, J., \& Valcarce Sánchez, R. (2016). Cuestionarios de actividad física para niños y adolescentes españoles: una revisión sistemática. An. Sist. Sanit. Navar, 39, 5-9. https://doi.org/10.7334/psicothema2012.125

Mayorga-vega, D., Parra Saldías, M., \& Viciana, J. (2019). Condición física, actividad física, conducta sedentaria y predictores psicológicos en adolescentes chilenos: Diferencias por género. Cultura_Ciencia_Deporte, 14(42), 233-241. https://doi.org/10.12800/ccd.v14i42.1337

Phillips, L. R. S., Parfitt, G., \& Rowlands, A. V. (2013). Calibration of the GENEA accelerometer for assessment of physical activity intensity in children. Journal of Science and Medicine in Sport, 16(2), 124-128. https://doi.org/10.1016/j.jsams.2012.05.013

Schaefer, C., Nigg, C., Hill, J., Lois, B., \& Browning, R. (2014). Establishing and Evaluating Wrist Cutpoints for the GENEActiv Accelerometer in Youth. Med Sci Sports Exerc, 46(4), 826-833. https://doi.org/ https://doi.org/10.1249/MSS.0000000000000150

Sirard, J. R., \& Pate, R. R. (2001). Physical activity assessment in children and adolescents. Sports Medicine (Auckland, N.Z.), 31(6), 439-54. https://doi.org/https://doi.org/10.2165/00007256-20013106000004

Stults-Kolehmainen, M. A., \& Sinha, R. (2014). The Effects of Stress on Physical Activity and Exercise. Sports Medicine, 44(1), 81-121. https://doi.org/10.1007/s40279-013-0090-5

Warren, T. Y., Barry, V., Hooker, S. P., Sui, X., Church, T. S., \& Blair, S. N. (2010). Sedentary Behaviors Increase Risk of Cardiovascular Disease Mortality in Men. Medicine \& Science in Sports \& Exercise, 42(5), 879-885. https://doi.org/10.1249/MSS.0b013e3181c3aa7e 